\title{
Study of Screen Printed Wells in Solid-State Ion Selective Electrodes
}

\author{
R. W. Hower and R. B. Brown \\ Center for integrated circuits and sensors, University of Michigan, Ann Arbor, MI 48109
}

\author{
E. Malinowska \\ Department of Analytical Chemistry, Warsaw \\ University of Technology, Warsaw, Poland
}

\begin{abstract}
The size of integrated ion sensors is dictated by the size and spacing of their polymeric membranes, which provide the chemical selectivity. The area occupied by an array of these membranes can be significantly reduced through the use of wells (areas separated by barrier walls, into which the membrane solutions are deposited). Screen-printable epoxies are obvious candidates for forming the wells, but their physical and chemical compatibility with polymer-based membranes has not been known. This paper describes a methodology for selecting a screen-printed epoxy well material, and compares 5 perspective materials.
\end{abstract}

\section{INTRODUCTION}

The size (and therefore cost) of solid-state ionselective sensors is usually determined by membrane dimensions and spacing, rather than by the size of circuitry, interconnects, or bonding pads. Membrane design rules are typically dictated by the requirement to keep membranes which are selective to different chemicals from touching. We have developed membranes for automated deposition by both screen printing [1] and dispensing. In both cases, membrane components are dissolved in solvents which are evaporated subsequent to deposition. The design rules must allow for flow-out of the membrane/solvent solution after it is applied to the sensor surface, making the sensors much larger than they would otherwise need to be.

To reduce the size of sensor arrays, we pattern wells (see Figure 1) which limit the flow-out of the membrane components, allowing membranes to be smaller and closer together. Wells provide the additional advantage of making

\author{
R. K. Meruva and M. E. Meyerhoff \\ Department of Chemistry, University of Michigan, \\ Ann Arbor, MI 48109
}

final membrane thickness more uniform, and the deposition process more tolerant of variations in the viscosity of membrane solutions. We and others have previously used epoxies [2,3], acrylic photopolymers [2], polyimide $[2,4]$, and silicon [5], to form wells or cavities. This work focuses on screen printing of epoxy wells, which can be a reliable and economical approach for forming wells if the characteristics of the epoxy are acceptable.

In this paper we report details of our screen-printed epoxy well process, and present the methodology for selecting the best material. Data will be presented which compares prospective materials based on: their chemical compatibility with the ion-selective membranes; adhesion of the well materials to the sensor surface (which is typically excellent); adhesion of the membranes to the well materials; and screen pnntıng characteristics (viscosity and thixotropy). The most important material property is chemical compatibility with the membrane, which means that the well material will not exude contaminants that interfere with the selectivity of the membrane, nor absorb active components from the membrane, reducing over time the magnitude of the sensor's response. Good adhesion of the membrane to the well is necessary to prevent electrolytic shunts around the membrane. When sensors have internal electrolyte layers, adhesion of the membrane to the well becomes even more important because osmotic pressure works to dislodge the membrane, and the area of adhesive contact between the membrane and sensor surface is reduced by the area of the internal layer.

Well materials for this study were selected for their screen printability and adhesion to silicon nitride. Screen masks were designed to help optimize the well dimensions.

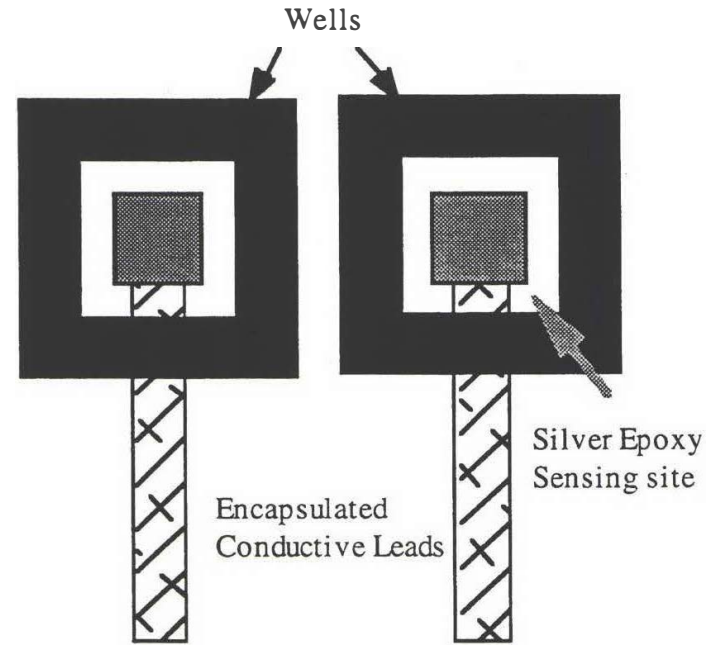

a)

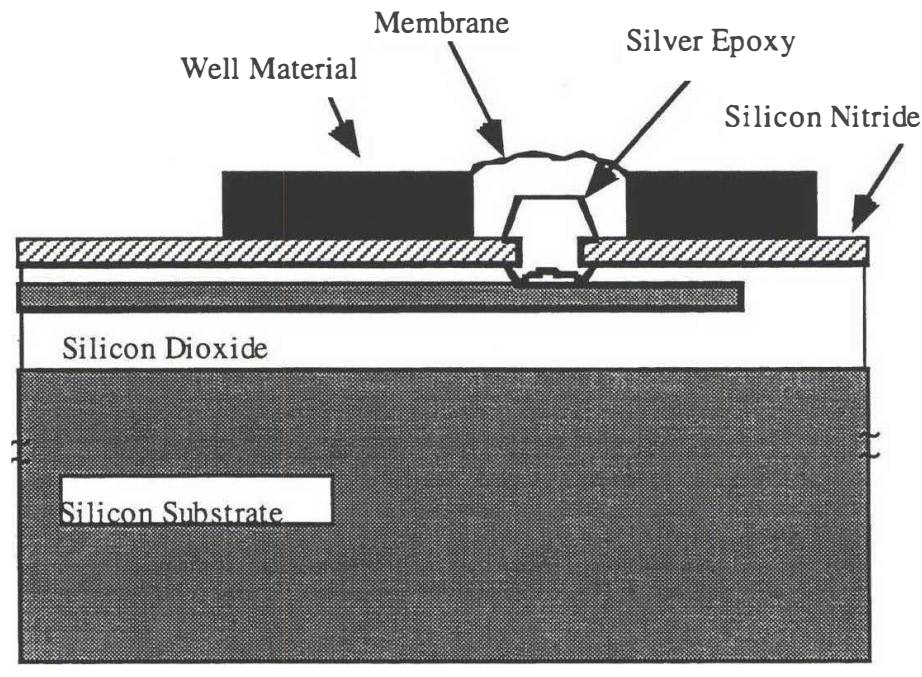

b)

Figure 1. The well surrounding the ion-selective electrode sensing site: a) top view, b) side view. 


\section{EXPERIMENTAL}

Reagents: Tecoflex polyurethane SG-80A (PU) was purchased from Thermedics (Woburn, MA). A terpolymer denoted as PVA (MW 40,000), which is a mixture of poly(vinyl-chloride)(80\%), poly(vinyl-acetate) (15\%) and poly(hydroxy propyl acrylate) (5\%), was purchased from Scientific Polymer Products, Inc. (Ontario, NY). The plasticizer dioctyladipate (DOA) was purchased from Scientific Polymer Products, Inc. (Ontario, NY).. The well materials used for this study were 688-PFC and 905 from Epoxy Technologies, Inc. (Billerica, MA), 3140 Silicon Rubber from Dow Corning (Midland, MI), EP3HT from Master Bond, Inc. (Hackensack, NJ), and BA-2116 from Tra-Con (Medford, MA).

Adhesion: The adhesion study was conducted by screen printing an unpatterned layer of each prospective well material onto silicon wafers, the surface of which had been coated with a silicon nitride layer. The well materials were cured, as appropriate for each material, and a scribe mark was made by sawing the wafer part way through. Membranes for the adhesion tests were formed by combining $1.06 \mathrm{~g}$ of DOA with $0.54 \mathrm{~g}$ of PU/PVA $(4: 1)$ to which was added $10 \mathrm{ml}$ freshly distilled Tetrahydrofuran (THF) to dissolve the components. After 5 hours of mixing, approximately $7 \mathrm{ml}$ of THF was evaporated from the solution under nitrogen, resulting in a slightly viscous solution. $500 \mathrm{ml}$ of this membrane cocktail was dispensed into a 2-cm square Teflon ring, centered on the wafer's scribe lane, on the surface of the cured well material. The membranes were allowed to cure for 24 hours before the ring was removed to allow the remaining THF to evaporate. The samples were cleaved on the scribe lane, and the membranes were pulled on a Q-Test II custom adhesion tester at $1 \mathrm{~cm} / \mathrm{min}$, at approximately 90 degrees angle from one side of the wafer. Five or six samples were pulled for each well material, and averages and standard deviations were compared with those of control samples, which had membranes in direct contact with the silicon nitride.

Membrane contamination: To study the chemical compatibility of the various materials to polymeric membranes, samples of each prospective well material were cast on Teflon and cured according to their normal cure schedules. $1.0 \mathrm{~g}$ of the cured material was removed from the Teflon and placed in a container with $4 \mathrm{ml}$ freshly distilled THF. The well material and THF were agitated for 24 hours, followed by the addition of $4 \mathrm{ml}$ THF and agitation for another 24 hours. The remaining solids were removed from the THF, and it was used in the preparation of test membranes, which were mounted in conventional Phillips electrode bodies. The THF contained the extractable contaminants from the well material, which were concentrated in the resulting membranes by evaporating the solvent. This is an extreme test of chemical compatibility.

Blank standard membranes contained 33 wt.\% PU/PVA (4:1) and $67 \mathrm{wt} . \%$ DOS. The first set of sodium membranes contain 1 wt.\% Monensin Methyl Ester (MME), $30 \mathrm{~mol}-\% \mathrm{KTpCIPB}, 32-33$ wt.\% PU/PVA (4:1) and 65-67 wt.\% DOS. In later membranes, the MME was increased to 4 wt.\%, with the other components decreased proportionally. Borates were used because they have been shown to decrease the anionic response in cationic selective membranes $[6,7]$. 3140 RTV Silicone Rubber was not tested because it is known to be chemically pure enough to form ion-selective membranes [8].

Screen-printing: The wells were printed using a $50-\mu \mathrm{m}-$ thick, $25-\mu \mathrm{m}$-mesh, screen mask. Wells were formed in a variety of sizes ranging from $0.75 \mathrm{~mm}$ to $1.15 \mathrm{~mm}$. The wells were screen printed on wafers having $50-\mu \mathrm{m}$-high, 300$\mu \mathrm{m}$-square, silver epoxy contacts centered in the wells.

Design rules: The optimal well design rules (spacing of well from silver epoxy contact and minimum wall thickness) were determined by examining screen-printed membranes in wells of various sizes. Ion-selective membranes were screen printed into the wells through $0.75 \mathrm{~mm}$ square openings in a $150-\mu \mathrm{m}$-thick stencil mask. The membranes were examined under a microscope to determine the integrity of the well walls, and whether the wells were completely filled. They were cleaved and examined with a scanning electron microscope (SEM) to evaluate the shape of the printed membranes.

\section{RESULTS}

Results of the adhesion, membrane compatibility, and screen printing experiments are shown in Table 1. Our PU/PVA membranes adhered satisfactorily to all of the well materials tested except 3140 RTV Silicone Rubber. The best adhesion was provided by the EP3HT and the 688-PFC epoxies.

Some of the blank (no ionophore) membranes and sodium membranes having little ionophore, showed anionic response; results for the blank membranes are shown in Figure 2. The top curve in this figure is for the control

\begin{tabular}{|l|c|c|c|c|c|}
\hline & \multicolumn{2}{|c|}{ Adhesion } & \multicolumn{2}{c|}{ Chemical } & \\
\hline $\begin{array}{c}\text { Well Material (number of adhesion } \\
\text { samples) }\end{array}$ & $\begin{array}{c}\text { Average } \\
(\mathrm{N})\end{array}$ & Std. deviation & Membrane Contamination. & Detection Limit & $\begin{array}{c}\text { Screen } \\
\text { printability }\end{array}$ \\
\hline Control (5) & 1.99 & 0.31 & none & $10^{-4}$ & \\
\hline Tra-Con BA-2116 (6) & 0.56 & 0.12 & some anionic & $10^{-3}$ & 1 \\
\hline Master Bond EP3HT (5) & 2.01 & 0.17 & none & $3 \times 10^{-4}$ & 1 \\
\hline Epo-Tek 688-PFC (5) & 1.89 & 0.16 & some anionic & $10^{-3}$ & 1 \\
\hline Epo-Tek 905 (6) & 1.29 & 0.30 & some anionic & no response & 2 \\
\hline 3140 RTV Silicon Rubber (6) & 0.15 & 0.11 & none & $10^{-4}$ & 3 \\
\hline
\end{tabular}

Table 1. Experimental results for studies of adhesion, chemical contamination, and screen printability. The detection limit is for sodium selective membrane with increased ionophore. The control for the adhesion test was silicon nitride, and for the chemical tests was a membrane containing distilled THF. 


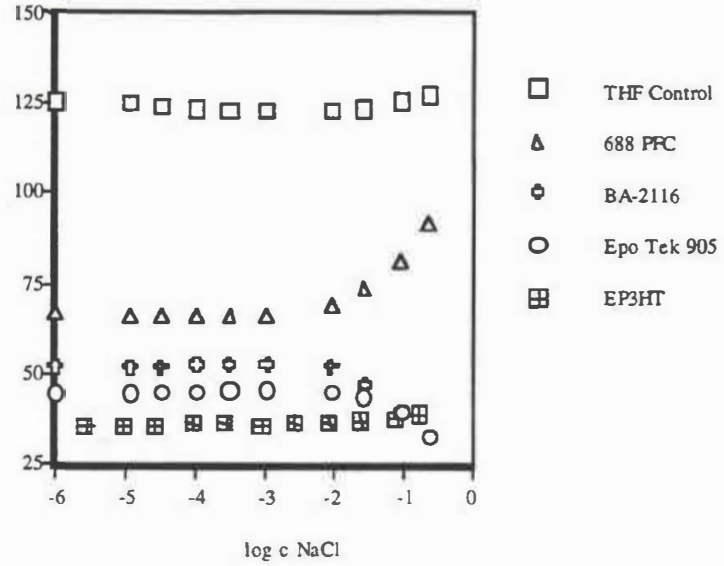

Figure 2. Anionic contamination of blank membranes in a calibration solution of $0.1 \mathrm{M} \mathrm{CaCl2}$. The top curve is for a standard blank (no ionophore) membrane; the lower curves are for blank membranes with extracted contaminants from the materials noted in the legends.

membrane, which was made with pure (uncontaminated) THF. The contaminated membranes (bottom three curves) have significantly lower baseline potentials, and respond to anions at high concentrations. This effect can be overcome in most membranes by adding more ionophore (e.g., 4 wt.\% instead of $1 \mathrm{wt} . \%)$. Figure 3 demonstrates this for sodium membranes; here the contamination has only increased the detection limit.

Screen printability was good to excellent, as expected, for all the well materials, as they were selected for this property. Table 1 lists a subjective evaluation of the printability of each material ( 1 is excellent, 5 is unacceptable). While silicone rubber has a number of very good properties from a screen printing point of view, it was given a fair rating because of the difficulty in cleaning it from the screen. Special solvents and mechanical action were needed to remove it from the screen, while the other well materials could be cleaned in acetone and isopropyl alcohol.

The relationship between well dimensions and mask opening for a particular membrane material is important to

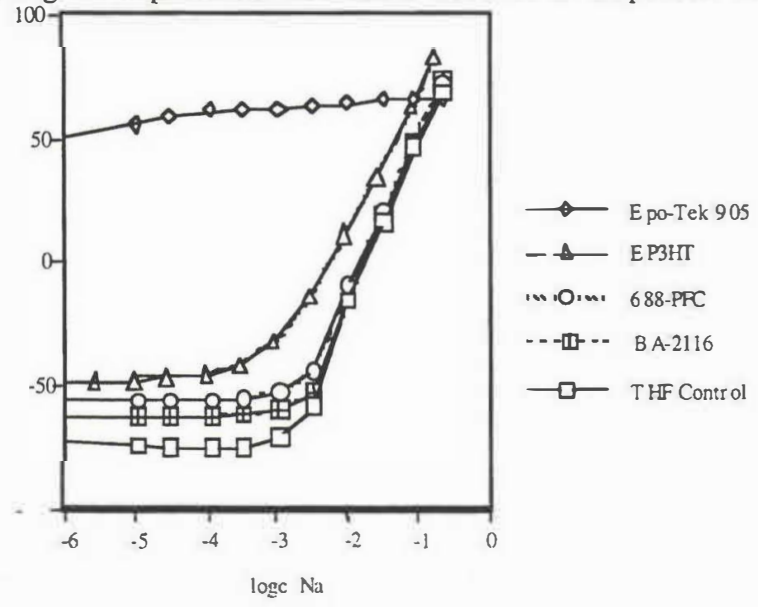

Figure 3. Improved sodium response by increasing the ionophore and borates to $4 \mathrm{wt}$. \%.

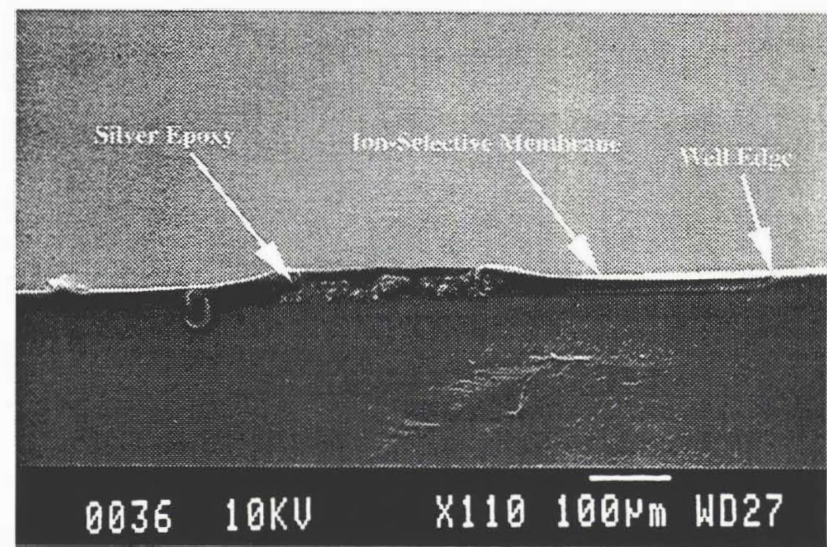

Figure 4. SEM of lon selective membrane printed into a EP3Ht well.

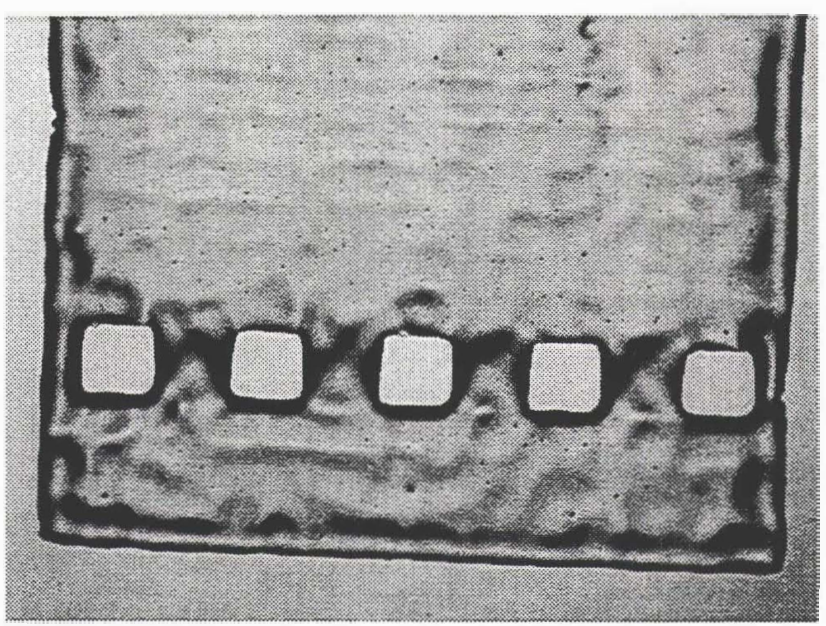

Figure 5. Picture of screen printed 3140 RTV silicone rubber wells.

successful use of the well concept. If the well is too small, membrane solution will overflow it, and may contaminate another membrane. If the well is too large, membrane thickness and dimensions will be more sensitive to viscosity of the membrane solution, and membranes will not be as uniform (as in the case of using no wells). Figure 4 is a SEM micrograph of a properly printed well. Figures 5 and 6 are pictures of screen-printed wells. We found that for our PU/PVA membranes, the well should be $200-400 \mu \mathrm{m}$ larger on a side than the opening in the membrane mask. When silver epoxy contacts partially fill the well, the membrane mask opening must be reduced in size. When printing over the silver epoxy bumps, it was observed that the well size was $100 \mu \mathrm{m}$ smaller than the drawn width. This needs to be taken into concideration when designing the wells.

\section{SUMMARY}

Thick-film wells on solid-state liquid chemical sensor surfaces facilitate the manufacturing process by making it less sensitive to the viscosity and surface-tension properties of the membrane solution. Properly-sized wells control the thickness and lateral dimensions of the 


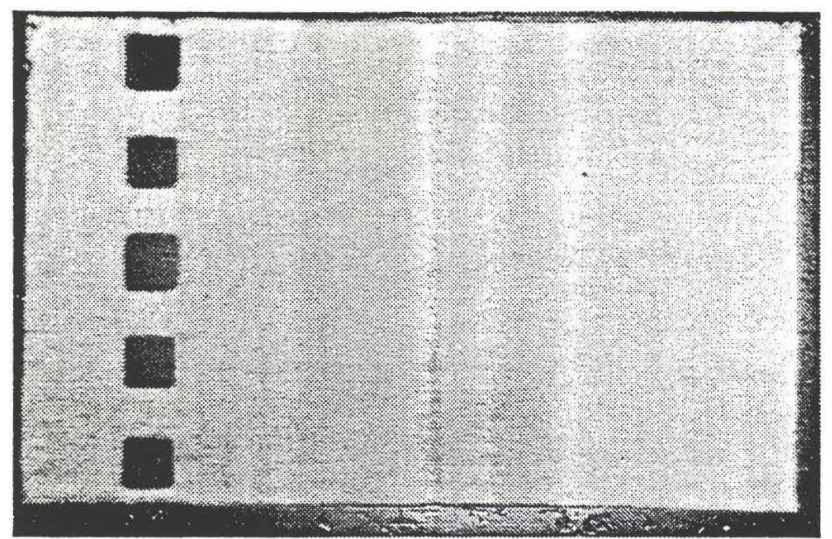

Figure 6. Picture of screen printed 688-PFC wells.

membranes. Wells allow closer membrane spacing, and result in more uniform sensors.

Materials must be evaluated in three general areas to determine their appropriateness for use as membrane wells. First, membrane compatibility must be determined to see if the material would contaminate the membrane. Second, adhesion tests must be done to determine if the material is chemically compatible with the membrane material. Finally, the screen-printing properties (primarily viscosity and thixotropy) must be examined by actually printing wells.

In this study, four epoxies and a silicone rubber were evaluated. The results showed that two of the epoxies have all of the properties needed for use as wells. The first is Epo-Tek 688-PFC, which has excellent screen-printability, and excellent adhesion to both the silicon nitride surface and to the ion-selective membrane. It showed some anionic response due to contaminants which might leach into the membrane, but even in the extreme case that was evaluated (extraction of contaminants with THF), this anionic sensitivity was overcome by increasing the amount of ionophore in the membrane. The second epoxy is the Master Bond EP3HT, which also has excellent screen printability and has excellent adhesion to the silicon nitride and the membrane. This material showed no anionic response in the contaminated membrane, and had very little change in the detection limit.

\section{REFERENCES}

1. R. W. Hower, J. H. Shin, G. S. Cha, R. K. Meruva, M. E. Meyerhoff, and R. B. Brown, "New Solvent System for the Improved Electrochemical Performance of Screen-Printed Polyurethane Membrane-based Solid-State Sensors.", Proceedings of Transducers 95 / Eurosensors IX, Stockholm, Sweden, June 1995, pp. 858-862.

2. R. B. Brown, An Integrated Multiple-Sensor Chemical Transducer, Ph. D. Dissertation. L'niversity of Utah, 1985.

3. H. D. Goldberg, The Batch Fabrication of Integrated Chemical Sensor Arrays, Ph. D. Dissertation, University of Michigan, 1993.

4. S. N. Cozzette, G. Davis, J. A. Itak, I. R. Lauks, R. M. Mier, S. Piznik, N. Smit, S. J. Steiner, P. Van Der Werf, and H. J. Wieck, "Wholly Microfabricated Biosensors and
Process for the Manufacture and Use Thereof.", U. S. Patent, 5,200,051, May 18, 1993.

5. L. J. Bousse, J. W. Parce, J. C. Owicki, K. M. Kercso, "Silicon micromachaning in the fabrication of biosensors using living cells.", Technical Digest. IEEE Solid-State Sensor and Actuator Workshop 173-6; Hilton Head Isl., SC, 6/90, Transducer Research Foundation, Cleveland (1990).

6. R. Eugster, P.M. Gehrig, W. E. Morf, U. E. Spichiger, W. Simon. "Selectivity-Modifying Influence of Anionic Sites in Neutral-Carrier-Based Membrane Electrodes.", Analytical Chemistry, vol. 63, pp. 2285-2289 (1991).

7. U. Schaller, E. Bakker, U. E. Spichiger, E. Pretsch, "Ionic Additives for Ion-Selective Electrodes Based on Electrically Charged Carriers.", Analytical Chemistry, vol. 66, pp. 391398 (1994).

8. E. Malinowska, V. Oklejas, R. W. Hower, R. B. Brown, and M. E. Meyerhoff, "Enhanced Electrochemical Performance of Solid-State Ion Sensors Based on Silicone Rubber Membranes.", Proceedings of Transducers 95 / Eurosensors IX, Stockholm, Sweden, June 1995. 